\title{
RETINOPATHY OF PREMATURITY
}

\section{RETINOPATÍA DE LA PREMATURIDAD}

\author{
BLANCO TEIJEIRO MJ ${ }^{1}$
}

Prematurity retinopathy (PR) is probably the area of Pediatric ophthalmology which arouses most interest at present. The rigorous clinical and basic research (good examples of the application of evidence-based medicine in our specialty) carried out in the last two decades has revolutionised our pathogenic knowledge. This has allowed the design of new and probably more effective therapeutic strategies based on the utilisation of antiangiogenic agents.

Prematurity retinopathy is the most frequent cause of infant blindness in developed countries. Although we do not have reliable data about its prevalence in Europe, it is known that in the United States every year between 14,000 and 16,500 premature babies are born weighing under $1,250 \mathrm{~g}$. Of these, between 9,000 and 10,500 develop some degree of PR. Between 1,000 and 1,500 premature newborns require treatment, and between 400 and 600 shall be legally blind. In addition, the seriousness of neonatal retinopathy constitutes a marker of late functional disability of these patients.

Prematurity retinopathy is a proliferative vascular disease affecting premature newborns and occurs during vessel development and maturation. It was described for the first time by Terry in 1940 as retro-lental fibroplasy, a cicatricial form of the disease. In the Fifties, it was related to a high and uncontrolled provision of oxygen, which led to a strict control thereof in Neonatology Units. This controlled administration of oxygen led to a marked reduction of the prevalence of PR, but the neonatal death rate increased due to respiratory and neurological complications. In the Eighties, the greater development of neonatal care techniques and methods has facilitated a greater survival rate of premature newborns with a lower gestation period $(<27$ weeks) and lower birth weights $(<1,000 \mathrm{~g})$, that is, premature newborns having a greater risk of PR. Although some studies indicated increases in the prevalence of more severe forms of PR, other recent studies point to a reduction of the prevalence, severity and progression of the disease in developed countries.

The International Classification of Prematurity Retinopathy (ICROP) described the degrees of PR severity on the basis of four parameters: localisation, stage, extension and presence of plus disease. Said classification, slightly modified and reviewed in 2005 (1), unified criteria and facilitated large multicentre clinical essays which contributed to improve our knowledge of the pathogeny of this disease.

At present we know that most of PRs regress; under $10 \%$ of PRs in stage 1 and 2 evolve to become threshold diseases. The Multicentre Study of Cryotherapy for Prematurity Retinopathy (CRYOROP) (2) defined the threshold disease (PR in stage three in zones 1 or 2 with plus disease, the extension of which is of five continuous or eight discontinuous hours) as the PRs level of severity where the risk of an unfavourable anatomic result was of $50 \%$. The threshold disease develops in approximately $6 \%$ of premature newborns weighing under $1,250 \mathrm{~g}$. The CRYO-ROP demonstrated the efficiency of peripheral cryotherapy for reducing unfavourable results both at the anatomic as well as the functional level. However, the percentage of unfavourable results observed in eyes with PR in zone 1 was high $(87 \%)$ in spite of the treatment. This led to the development of subsequent clinical studies [STOP-ROP (Therapeutic Oxygen Supplements for the Prevention of pre-Threshold Prematurity Retinopathy) and ET-ROP (Study of Early Treatment of Prematurity Retinopathy)] which defined and subdivided the pre-threshold disease as eyes with a high risk of

\footnotetext{
1 Ph.D. in Medicine. Ophthalmologist Service of the University Hospital Complex pf Santiago de Compostela (CHUS). Spain. E-mail: maria.jose.blanco.teijeiro@sergas.es
} 
developing the threshold disease and therefore should be closely followed. ET-ROP (3) states that the treatment of PR in earlier stages improves the visual and functional prognosis. At present, laser photocoagulation is utilised for ablation of the peripheral retina because its superiority vis-a-vis cryotherapy has been demonstrated.

From the numerous clinical essays we have derived the knowledge of the natural history of PR and the evidence that timely treatment reduces the risk of visual loss. Therefore, effective care of premature newborns requires careful exam at the right time and by an ophthalmologist with vast experience in examining these patients (the screening and followup indications have been published jointly by the American Ophthalmology and Pediatric Academies). On many occasions, pre-term newborns must be referred to reference ophthalmology centres for examination and treatment, thus increasing morbidity due to transport. By means of the acquisition of digital eye fundus images and their transmission to these specialised centres, telemedicine provides the opportunity of identifying patients at risk which require in situ exploration or treatment. Said digital images would also facilitate the development and execution of multicentre clinical essays. Finally, the photographic documentation of the treatment would allow us to differentiate between genuine therapeutic failure and bad results caused by incomplete treatments.

Due to the research carried out in animal models, it is known that PR is a two-stage disease (the first is a hyperoxic vessel obliteration and the second is a neovascularisation stage). The discovery of the importance of VEGF (vascular endotelial growth factor) and IGF-I (insulin-like growth factor-I) (4) in the development of PR constitutes an important step to understand the pathogeny of the disease. VEGF is necessary for the physiological angiogenesis, and it is regulated by tissue hypoxia. It has also been associated with vitreous neovascularisation in experimental models. IGF-I is an important somatic growth factor which correlates very well with the birth weight and gestational age. IGF-I is also a key factor in normal vessel development and regulates retinal neovascularisation by controlling the activation of VEGF regardless of the oxygen conditions. These findings suggest a number of alternatives for the medical treatment of the disease but also (and this is even more important) a critical moment for performing said intervention. The early inhibition of VEGF or IGF-1 after birth could avoid normal vessel growth and precipitate the disease, whereas the inhibition in the second stage could prevent neovascularisation. The finding that the latest development of PR is associated to lower levels of IGF-I (5) after premature labour suggests that is the physiological and early replacement of IGF-I at the levels found in the uterus could prevent the disease since it allows normal vessel development. On the contrary, if the IGF-I supplementation is performed late, in the neovascular phase, the disease would be exacerbated.

All the above points to a future in which, instead of emphasising the type of treatment, we would have to focus on the prevention of the disease by utilising anti-angiogenic agents acting at the molecular level. The perfect agent would be one which could be administered in the least invasive manner (eye drop or single intravitreous injection) and had the minimum effect on normal vascular development while preventing neovascularisation and yielding the lowest possible retinal toxicity.

\section{REFERENCES}

1. International Committee for the Classification of Retinopathy of Prematurity. The International Classification of Retinopathy of Prematurity revisited. Arch Ophthalmol 2005; 123: 991-999.

2. Cryotherapy for Retinopathy of Prematurity Cooperative Group. Multicenter Trial of Cryotherapy for Retinopathy of Prematurity: ophthalmological outcomes at 10 years. Arch Ophthalmol 2001; 119: 1110-1118.

3. Early Treatment For Retinopathy Of Prematurity Cooperative Group. Revised indications for the treatment of retinopathy of prematurity: results of the early treatment for retinopathy of prematurity randomized trial. Arch Ophthalmol 2003; 121: 1684-1694.

4. Hellstrom A, Perruzzi C, Ju M, Engstrom E, Hard AL, Lui $J L$, et al. Low IGF-I suppresses VEGF-survival signaling in retinal endothelial cells: direct correlation with clinical retinopathy of prematurity. Proc Natl Acad Sci USA 2001; 98: 5804-5808.

5. Hellstrom A, Engstrom E, Hard AL; Albertsson-Wikland $K$, Carlsson B, Niklasson A, et al. Postnatal serum insulinlike growth factor I deficiency is associated with retinopathy of prematurity and other complications of premature birth. Pediatrics 2003; 112: 1016-1020. 